\title{
Optical Tandem Side Band treated (OTSB)-FSO link under the influence of Turbulent Environment
}

\author{
[Vishal Sharma, Navneet Kaur Bajwa]
}

\begin{abstract}
The foremost challenge that FSO come across is the transmitting media linking transmitters and receivers where many weather conditions can influence the capacity of the FSO link such as level of rain, haze, Fog, cloud bursting, scattering and turbulence resulting in fading of the transmitted signal and causing high bit error rate or signal loss at the receiver end. In this work, the impact of turbulence on OFDM-OTSB-FSO system is modeled and analyzed using the gamma-gamma distribution modeling at 10 Gbps over the FSO link. To achieve high speed FSO link under the sway of small and high scale turbulence variations, OFDM-OTSB treated data transmission is reported and demonstrated.
\end{abstract}

Keywords-FSO, OFDM, OTSB, Turbulence

\section{Introduction}

Free Space Optical (FSO) communication provides a considerable possibility for producing a three-dimensional world-wide communications framework. Many of the features of FSO are associated to fiber optics with an essential distinction of communicating link which is air/free space rather than the glass of the fiber-cable. The extraordinary extension of internet and alternative wireless networks have a huge influence in today's world. Being in the era of ineradicable connectivity, the necessity of high speed networks have put the constraint on wireless communication networks to improve the transmission capacity of system and the coverage area of the signal. This is primarily due to the simplicity of installing FSO links, broad accessible bandwidth and unobstructed frequency band. Distinguished capacity, low power consumption, light weight, small sizes, high data rates and low costs for satellite cross-links are other promises of FSO technology. It is an important building block for wide area space networks thus assisting mobile users, high speed data services for small satellite terminals and serving as a mainstay network for high speed trunking [1].

\section{Vishal Sharma}

Shaheed Bhagat Singh State Technical Campus, Ferozepur, Punjab India

Navneet Kaur Bajwa

Shaheed Bhagat Singh State Technical Campus, Ferozepur, Punjab India
Despite all these benefits, FSO is very expensive stopping it from substituting the present wireless networks and these advantages are feasible only under ideal channel conditions while atmospheric occurrences such as clouds, fog, aerosols, and even turbulence can seriously demean the system performance. These disturbances have different degrading affects on signal travelling through the FSO link, while clouds and aerosols disperse the laser beam and cause optical pulse attenuation and broadening in space and time, turbulence reveal itself as disturbances in the amplitude and phase of received optical signal.

Most likely known weather occurrences are scattering and turbulence resulting in fading of the transmitted signal and causing high bit error rate or signal loss at the receiver end. Atmospheric turbulence is a phenomenon which comes into sight when there are fluctuations in the refractive index because of in-homogeneity in temperature and pressure conditions. Hence, atmospheric turbulent channel is hugely erratic, irresolute and unshielded to weather effect generating variations in intensity and phase of the received signal because of instability in the refractive index through the propagation path. As a result, atmospheric turbulence can provoke severe error rates due to the random fluctuations in the received signal. Among the other challenges faced by a turbulent FSO link, power loss of the optical signal is one of the main challenges that affect the capacity of system especially at high data rates since it results from losses present at the transmitter and receiver side of the system. The FSO transmission is also highly affected by multipath fading. A notable attempt is made to design a long reach multi-carrier FSO system free from the impact of multipath fading using OSSB modulation scheme. An OFDM-OTSB-FSO direct detection system is mentioned to be the optimal FSO system to provide a continuous FSO link with adequate BER [2]. Further, the work is extended to analyze the performance of W-OFDM systems which illustrates the outperformance of orthogonality of W-OFDM sub-carrier weigh against C-OFDM [3]. A number of recent studies show the advancement in researches involving OFDMFSO systems [6-9]. A study also revealed the preference of OFDM-IsOWC systems over conventional IsOWC systems so as to improve the BER at $5 \mathrm{Gbps}$ to provide prolonged ISL link between two satellites [2]. This paper extended the previous related work to compute the performance of OFDM- 
OTSB-FSO system under turbulent conditions at 10Gbps and the influence of signal power loss during transmission along with transmitter and receiver loss. The remainder of this paper is organized as follows. Section II presents a concise theory of atmospheric fluctuations along with the simulated test bed. Section III is covers the results and discussions for the obtained observations and is followed the conclusion drawn from the obtained simulative results in Section IV.

\section{System Modelling}

An OFDM-OTSB-FSO system is modeled under various turbulent conditions of the atmosphere. The OFDM modulated signal at $10 \mathrm{Gbps}$ with deviating signal power loss along with transmitter and receiver loss are transmitted through the FSO channel. A SNR of $20 \mathrm{~dB}$ is allocated to be a passable bound for a satisfactory performance of the system. The FSO system comprise of three constituent communication components which are: transmitter, propagation link and receiver. The FSO system is not much variant from fiber optic communication as the difference relies mostly in the propagation link. The transmitter comprises of a pulse generator, $\mathrm{CW}$ laser, Mach Zehnder modulator, optical filter and an amplifier. At the transmitting terminal, a 4-QAM sequence generator produces a data signal with 2 bit per symbol. This data is then OFDM modulated by an OFDM modulator using 512 subcarriers. OFDM analog signals are produces by taking the FFT size as 1024 which are then QAM modulated at a modulator frequency of $7.5 \mathrm{GHz}$. These OFDM analog data signals are then communicated over FSO channel by deploying a preferable OTSB technique rather than deploying ODSB technique as it is immensely prone to fading. OTSB modulation in this case is undertaken by a dual electrode Mach Zehnder modulator (DEMZM) and a phase shifter. The receiver end of the FSO system comprises of a photo detector and a low pass filter to retrieve high rate OFDM data effectively. This signal is successfully de-spread through a direct detection method used at the receiver terminal to retrieve the signal completely. Two telescopes having a FSO link between them make up the free space channel with beam divergence of $2 \mathrm{mrad}$. The length of the FSO link is spread up to $10 \mathrm{~km}$ to determine the performance of the channel under turbulent environment. The evaluated attenuation of free space between the connecting FSO nodes is observed to have a consistent value of $5 \mathrm{~dB}$. The additional losses are assumed to be $0 \mathrm{~dB}$ and the propagation delay is $0 \mathrm{ps} / \mathrm{km}$. The signal power loss is considered to be either $0 \mathrm{~dB}$ or $-3 \mathrm{~dB}$. The transmitter loss is taken to be either $5 \mathrm{~dB}$ or $10 \mathrm{~dB}$ while the receiver loss is taken either $10 \mathrm{~dB}$ or $20 \mathrm{~dB}$.

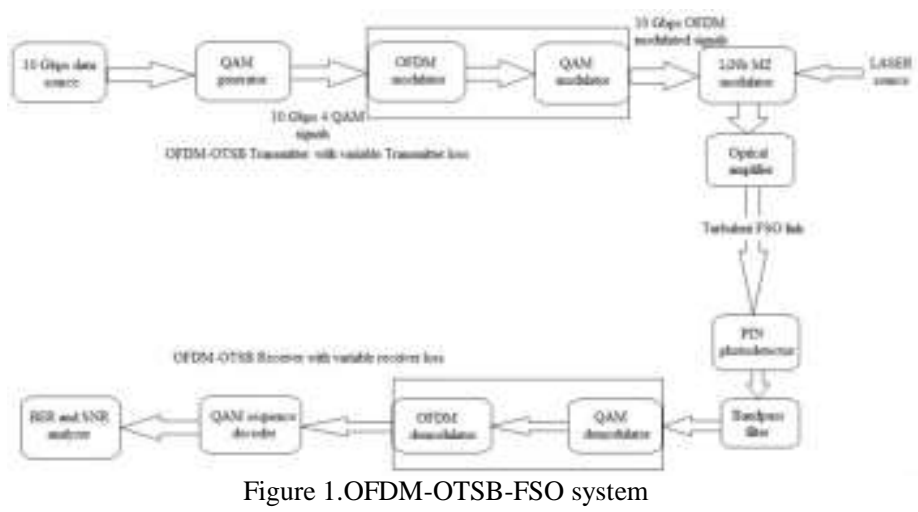

\section{Results and Discussion}

The assessment of SNR of an OFDM-OTSB-FSO signal under various turbulent surroundings is reported. The value of signal power loss which serves as an important parameter relating to the impact of turbulent behavior is taken into account. At the transmitter terminal of the simulated test-bed, a 10Gbps signal is transmitted over the FSO turbulent channel with OTSB scheme through a phase shifter and DEMZM. As it is apparent from Figures 1-4, OFDM-OTSB-FSO executes effectively within the range of $10 \mathrm{~km}$ for the accounted data rate of $10 \mathrm{Gbps}$ under the effect of atmospheric attenuation of 5 $\mathrm{dB}$. For a moderate turbulent channel, considering the power loss $=0 \mathrm{~dB}$ along with transmitter loss $=15 \mathrm{~dB}$ and receiver loss $=20 \mathrm{~dB}$, the FSO link is limited to $9 \mathrm{~km}$ with an acceptable SNR measured as shown in Figure 2. For power loss $=-3 \mathrm{~dB}$, the performance is noticed to be unfavourable beyond $8 \mathrm{~km}$, deteriorates drastically, and fails to provide an acceptable performance beyond FSO link of $8 \mathrm{Km}$. On considering the power loss $=-3 \mathrm{~dB}$, the system performance decreases as shown in Figure 3. Consequently, it is revealed that the signal power loss along with the transmitter and receiver loss are a significant aspect to improve FSO link at high data rate under the impact of turbulence conditions. Further, the simulated system is observed under strong turbulent conditions. It is observed that FSO link is limited due to a number of factors for instance power loss along with the transmitter and receiver loss along with the amount of fading caused by turbulence considering small scale- and large scale- atmospheric fluctuations as depicted in Figure 4-5. Considering the power loss $=0 \mathrm{~dB}$ along with transmitter loss $=5 \mathrm{~dB}$ and receiver loss $=10 \mathrm{~dB}$, the FSO link is limited to 7 $\mathrm{km}$ with an acceptable SNR measured as shown in Figure 4. For power loss $=-3 \mathrm{~dB}$, the performance is noticed to be detrimental beyond $6 \mathrm{~km}$. On considering the power loss $=-3$ $\mathrm{dB}$, the system performance decreases up to $6 \mathrm{~km}$ and declines acutely beyond $7 \mathrm{~km}$ as shown in Figure 5 . 
Proc. of the Second Intl. Conf. on Advances in Information Processing and Communication Technology - IPCT 2015

Copyright $\odot$ Institute of Research Engineers and Doctors, USA .All rights reserved.

ISBN: 978-1-63248-044-6 doi: 10.15224/ 978-1-63248-044-6-22

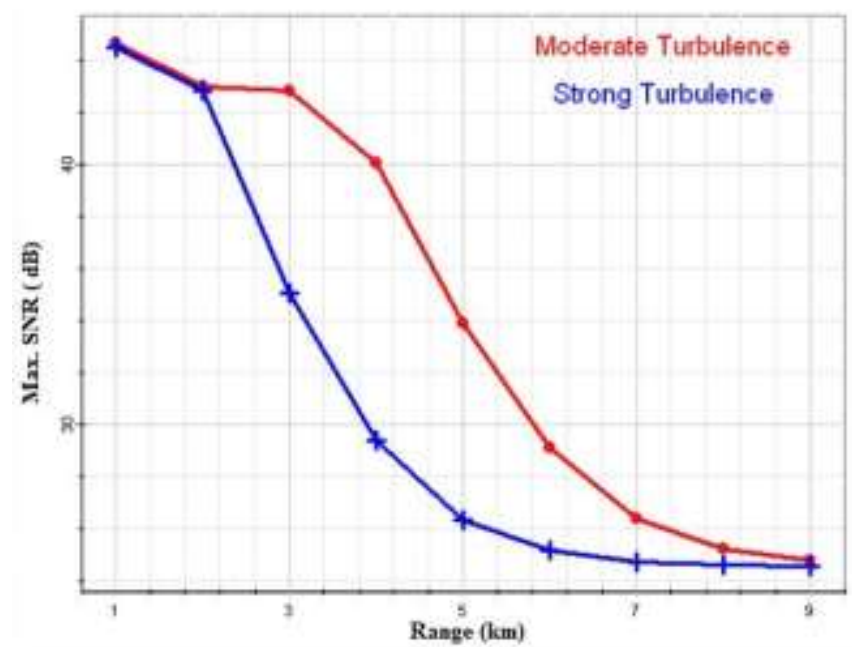

Figure 2. Evaluation of SNR of OFDM-OTSB-FSO at Power loss $=0 \mathrm{~dB}$, Transmitter loss $=15 \mathrm{~dB}$, Receiver loss $=20 \mathrm{~dB}$

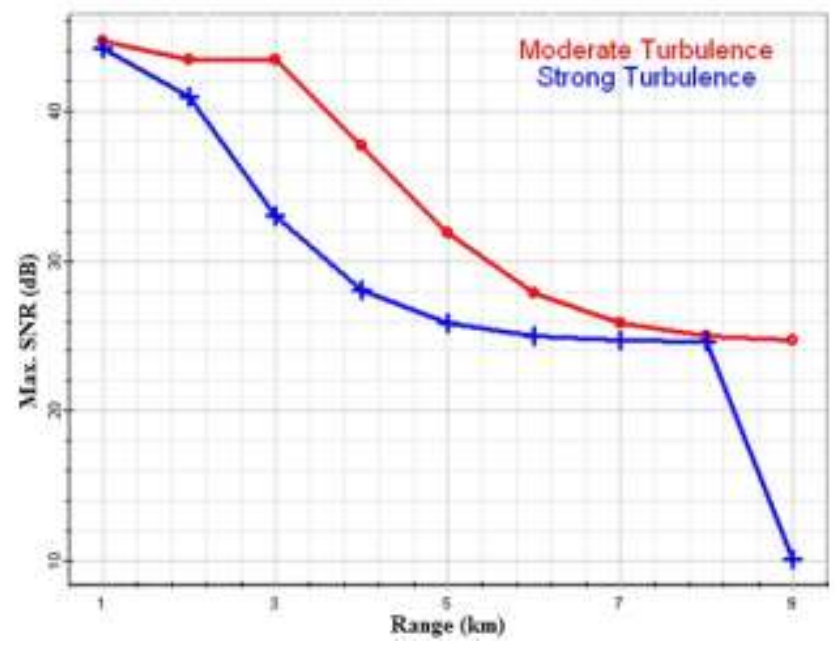

Figure 3. Evaluation of SNR of OFDM-OSSB-FSO at Power loss $=-3 \mathrm{~dB}$, Transmitter loss $=15 \mathrm{~dB}$, Receiver loss $=20 \mathrm{~dB}$

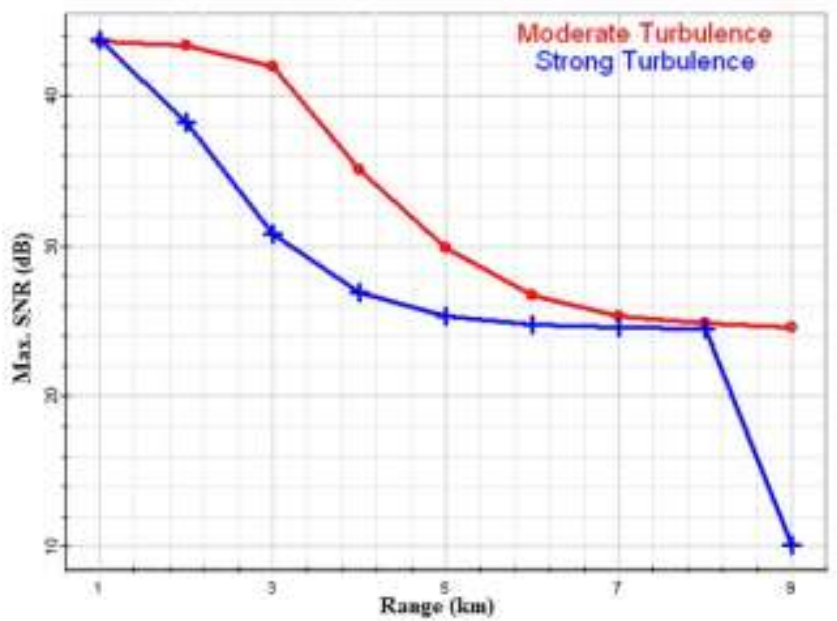

Figure 4: Evaluation of SNR of OFDM-OSSB-FSO at Power loss $=0 \mathrm{~dB}$, Transmitter loss $=5 \mathrm{~dB}$, Receiver loss $=10 \mathrm{~dB}$

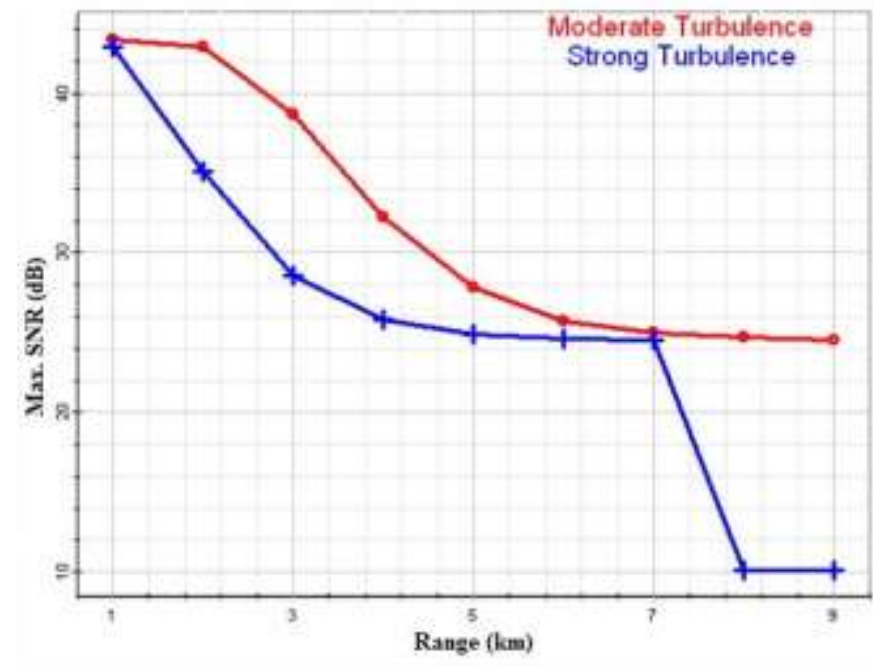

Figure 5: Evaluation of SNR of OFDM-OSSB-FSO at Power loss $=-3 \mathrm{~dB}$, Transmitter loss $=5 \mathrm{~dB}$, Receiver loss $=10 \mathrm{~dB}$

\section{Conclusion}

In this work, the impact of turbulence on OFDM-OTSBFSO system is analysed using the gamma-gamma distribution modelling at $10 \mathrm{Gbps}$ over the FSO link. It is noticed that FSO link is limited due to a number of factors for instance transmitter and receiver loss along with the amount of fading caused by turbulence considering small scale- and large scale- atmospheric variations. To achieve high speed FSO link under such conditions, OFDM and OTSB treated data transmission is recommended at low transmitter and receiver loss.

\section{References}

[1] Chan, V.W.S. "Optical space communications: a key building block for wide area space networks", Lasers and Electro-Optics Society, volume 1, pp: 41-42, 1999.

[2] Vishal Sharma, Gurimandeep Kaur, "High Speed, Long Reach OFDM-FSO Transmission Link Incorporating OSSB and OTSB Schemes", Optik Elsevier 124 (2013).

[3] Vishal Sharma, Amandeep Kaur, "Modeling and simulation of FBG based OCDMA IsOWC system", Optik, Elsevier 125 (2014).

[4] JC Ricklin, FM Davidson, "Atmospheric turbulence effects on a partially coherent Gaussian beam: implications for free space laser communication", J Opt Soc Am A 2002; 19(9):1794-802.

[5] Yixin Zhang, Congfang Si, Yuanguang Wang, Jianyu Wang, Jianjun Jia, "Capacity for non-Kolmogorov turbulent optical links with beam wander and 
Proc. of the Second Intl. Conf. on Advances in Information Processing and Communication Technology - IPCT 2015

Copyright $\odot$ Institute of Research Engineers and Doctors, USA .All rights reserved.

ISBN: 978-1-63248-044-6 doi: 10.15224/ 978-1-63248-044-6-22

pointing errors", Optics and Laser technology, Elsevier 43 (2011).

[6] V. Sharma, G. Kaur, "Modelling of OFDM-ODSBFSO transmission system under different weather conditions", IEEE Third International Conference on Advance Computing and Communication Technologies (ACCT 2013), 2013, pp.154-157.

[7] Vishal Sharma, Mini Lumba, Gurimandeep Kaur, "Severe climate sway in coherent CDMA-OSSBFSO transmission system", Optik, Elsevier 125 (2014).

[8] Vishal Sharma, Gurpreet Singh, "On BER assessment of conventional- and wavelet-OFDM over AWGN channel", Optik, Elsevier 125 (2014).

[9] Vishal Sharma, Amandeep Kaur, "Modeling and simulation of FBG based OCDMA IsOWC system", Optik, Elsevier 125 (2014).

[10] Vishal Sharma, Gurimandeep Kaur, "High Speed, Long Reach OFDM-FSO Transmission Link Incorporating OSSB and OTSB Schemes", Optik Elsevier 124 (2013). 